\title{
DOES A COMMON DYNAMO MECHANISM EXIST FOR LOWER MAIN SEQUENCE STARS?
}

\author{
R.B. TEPLITSKAYA and V.G. SKOCHILOV \\ SibIZMIR \\ P.O.Box 4, Irkutsk 33 \\ 664033, USSR
}

\begin{abstract}
Based on an extended list of lower main sequence stars from Rutten (1987), the relation between chromospheric activity and Rossby number has been revised. The increased statistics changes the shape of the curve as compared with that of Noyes et al. (1984). The saturation at small Rossby numbers has disappeared. The dependence on Rossby number in the range of very large Rossby numbers has weakened. The standard deviation of the activity indices from the mean curve is about $40 \%$. This scatter of individual stars is not due to differences in the spectral type or age of the stars.
\end{abstract}

It is generally recognized that chromospheric and coronal activity of solar-type stars is closely related to stellar rotation. Since the paper of Noyes et al. (1984) this relation has even been used for evaluating rotation periods and ages of stars for which direct determinations of these parameters are difficult. The 'activity-rotation' relation for the lower part of the main sequence implies that there exists a common curve for stars of all spectral types, from $\mathrm{F}$ to $\mathrm{M}$, in the $R^{\prime}, R o^{(\alpha)}$ diagram. $R^{\prime}$ is the activity index (the ratio between the emission flux in a chromospheric line $F^{\prime}$ corrected for the contribution of photospheric emission, and the bolometric flux). $R o^{(\alpha)}$, the Rossby number, is a measure of the rotation.

$$
R o^{(\alpha)}=P / \tau_{c}^{(\alpha)},
$$

where $P$ is the period of rotation, and $\tau_{c}^{(\alpha)}$ is the convective turnover time with mixing-length parameter $\alpha=l / H$. The Rossby number is directly related to the dynamo number, so the fact that it is $R o$ rather than the period or velocity of rotation that controls the activity is convincing evidence for a common mechanism of atmospheric heating by magnetic fields generated by the process of dynamo action.

Since it is not obvious how to choose the optimum index to characterize chromospheric activity, we have tried to introduce the new index

$$
A_{H K}^{\prime}=F_{H K}^{\prime} / F_{H K}^{\prime b}=R_{H K}^{\prime} / R_{H K}^{\prime b} .
$$

$F_{H K}^{\prime b}$ and $R_{H K}^{\prime b}$ are the values of $F_{H K}^{\prime}$ and $R_{H K}^{\prime}$ on the basic curve with the same colour index $(B-V)$ as in the star considered. The flux $F_{H K}^{\prime}$ is measured in the emission reversals 
of the lines $\mathrm{H}$ and $\mathrm{K}$ of $\mathrm{Ca}$ II. The only possible advantage of $A_{H K}^{\prime}$ over $R_{H K}^{\prime}$ is that the former may be colour independent, while this is not the case for the latter. However, the colour independence of $A_{H K}^{\prime}$ is valid only under special circumstances.

Using data from Rutten (1987), we have considered 182 main-sequence stars of spectral classes F2 to M4.5 and constructed a $\log A_{H K}$ vs. $\log R o^{(\alpha)}$ diagram (Teplitskaya, 1989). The curve obtained resembles the curve of Noyes et al. (1984) in the sense that it includes all stars, regardless of their spectral type. As concerns its shape it shows interesting differences, however: (1) There are no indications of a saturation of the chromospheric activity at small Rossby numbers, i.e., in the region where because of the large values of $\tau_{c}^{(\alpha)}$ a large number of late-type stars occur. (2) At large Rossby numbers the index $A_{H K}^{\prime}$ randomly fluctuates around a constant mean value. In the range of large $R o^{(\alpha)}$ there are many stars with very thin convective envelopes, because $\tau_{c}^{(\alpha)}$ is small. (3) The parameter $\alpha=1.6$ instead of 2.0 in Noyes et al. (4) The scatter of some stars around a mean curve is greater than can be explained by random or systematic errors. The above-mentioned differences occur for stars with very thick and very thin convective envelopes, and may be due to individual features of the dynamo action in some stars.

The first question we have to answer is whether the differences may be attributed to the use of different activity indices, or if they are a consequence of the increased sample of stars (41 stars in Noyes et al. (1984), and 182 stars in Teplitskaya (1989)). To settle this matter we have repeated the investigation of Noyes et al. (1984), but using the 182 stars listed by Rutten (1987). Figure 1 shows plots of $\log R_{H K}^{\prime}$ vs. $\log \tilde{R} o^{(2.0)}$ and $\log A_{H K}^{\prime}$ vs. $\log \tilde{R} o^{(1.6)}$ $\left(\tilde{R} o^{(\alpha)}\right.$ being the Rossby number $R o^{(\alpha)}$ normalized to its value for $\left.(B-V)=0.63\right)$. The open circles represent averages in coordinates over 20 or 21 stars.

There are now no fundamental differences in the shapes of the two curves. The indications of saturation are very weak, much less than what was found by Noyes et al. (1984) for 41 stars. On both plots (diagrams to the left) one can observe a branch of constant activity at large Rossby numbers.

Apart from the usual Rossby numbers we have also used the numbers

$$
R o^{(\alpha) \prime}=R o^{(\alpha)} f(M),
$$

where $M$ is the stellar mass, $f(M)=\left(\alpha H / R_{c}\right)^{1 / 2}$, and $R_{c}$ and $H$ are the radius and the pressure scale height at the base of the convection zone (Durney and Robinson, 1982). Corresponding 'activity-rotation' curves are shown in the right-hand diagrams of Figure 1. Signs of saturation of the chromospheric activity have become even weaker. Besides, the application of the modified Rossby numbers has almost completely eliminated the branch of constant activity, except for the stars with the largest values of $R o^{(\alpha) \prime}$. Thus the chromospheric activity of most of the stars under investigation can, on the average, be approximated by the power laws

$$
\begin{array}{ll}
R_{H K}^{\prime}=1.89 \times 10^{-4}\left[\tilde{R} o^{(2.0)^{\prime}}\right]^{-0.683}, & S= \pm 38.7 \% \\
A_{H K}^{\prime}=1.26 \times 10^{-4}\left[\tilde{R} o^{(1.6)^{\prime}}\right]^{-0.745}, & S= \pm 41.9 \% .
\end{array}
$$

$S$ is the relative standard deviation of $R_{H K}^{\prime}$ or $A_{H K}^{\prime}$ from the mean curves, calculated as $(\bmod )^{-1} \sigma_{y}$, where $\sigma_{y}$ is the standard deviation along the ordinate axes of Figure 1 , and $\bmod =\log e \approx 0.43429$. 
$\lg R_{H K}^{\prime}(I)$

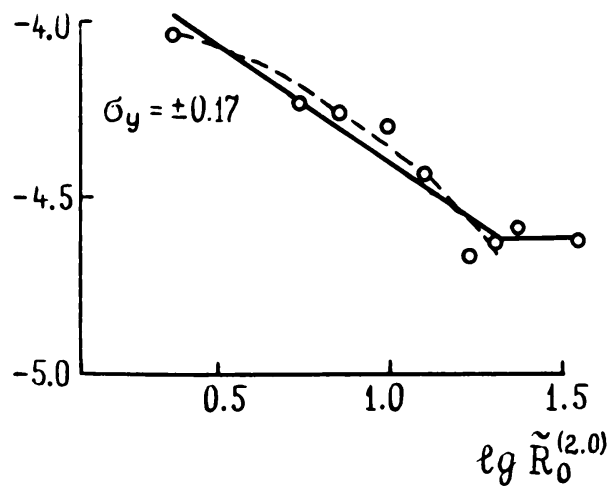

$\lg A_{H K}^{\prime}$ a

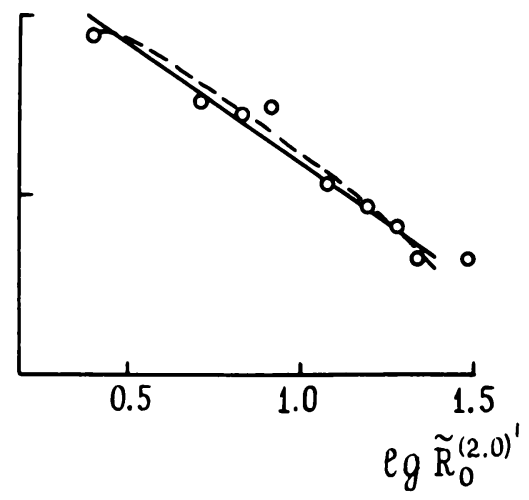

b

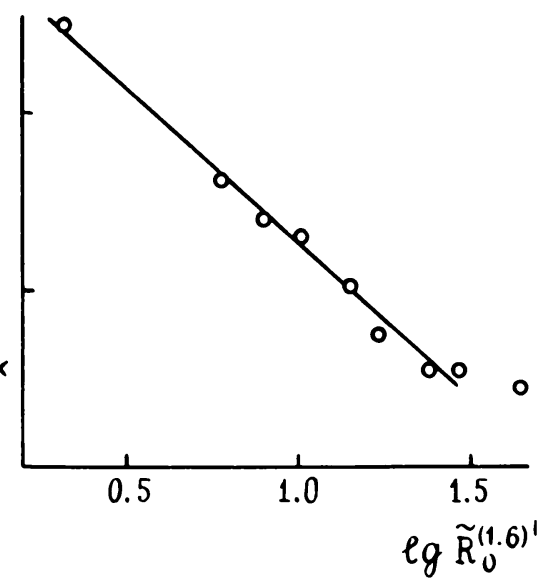

Figure 1. The 'activity-rotation' relation for two indices of chromospheric activity (a: $R_{H K}^{\prime}$, b: $\left.A_{H K}^{\prime}\right)$ and for two procedures of calculating the Rossby numbers (left and right diagrams).

The considerable scatter of the individual stars is uncorrelated with their colours. There is a slight dependence of the residuals (O-C) on the age of the star as shown in Figure 2. Estimates of the age of the stars and clusters have been taken from Duncan (1981), Catalano and Marilli (1983), and Barry et al. (1987). With the same colour and rotation period young stars tend to be slightly more active than old stars. Part of the scatter may be accounted for by the relative age, i.e., the different rate of evolution. For example, in stars of class $F$ the convective turnover time begins to change already during the main-sequence stage (Gilliland, 1985). Another example of a peculiar behaviour associated with age is that among the stars that have just arrived at the main sequence there are fast rotators, whose rotation velocities are not representative of their rather late spectral class K (Stauffer et al., 1984). However, these factors do not seem to be able to explain the main part of the scatter.

The absence of saturation in late-type stars at a first glance contradicts the concept of 


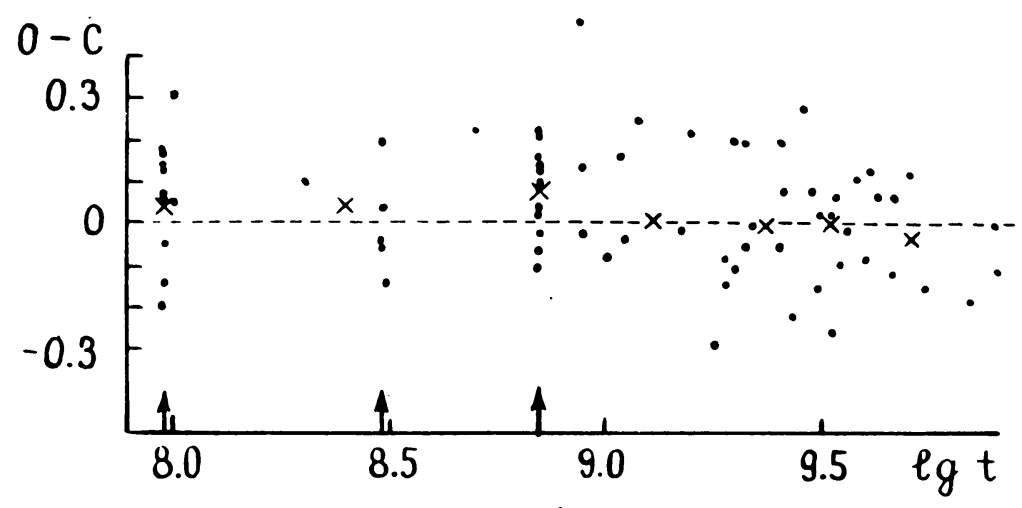

\section{Pleiades UMa Hyades}

Figure 2. Residuals with respect to the mean curves vs. stellar age. The dots represent individual stars, the crosses averages over 10 stars.

stabilization of the magnetic activity, arrived at from X-ray luminosity observations of $\mathrm{M}$ dwarfs. It seems likely that the behaviour of the chromospheric activity indices in lowmass stars is controlled not only by the efficiency of the dynamo mechanism but also, to a significant extent, by geometrical effects, i.e., very large filling factors of active regions, with overlaying magnetic 'canopies' as described by Giovanelli (1980) for the Sun, leading to excess heating of the chromosphere. In the case of very late $\mathrm{M}$ stars with $(B-V) \gtrsim 1.50$ Schrijver and Rutten (1987) found the opposite phenomenon, namely a deficiency of chromospheric emission as compared with X-ray emission. This results from a decrease of the role of ionized metal lines in favour of an increased role of the hydrogen lines for the radiative cooling of the chromosphere. In the sample we are investigating there are only two stars with $(B-V)>1.5$. Accordingly the deficiency caused by radiative transfer effects does not influence the results reported in the present paper.

\section{References}

Barry, D.C., Cromwell, R.H., and Hege, E.K. (1987) 'Chromospheric activity and ages of solar-type stars', Astrophys. J. 315, 264-272.

Catalano, S. and Marilli, E. (1983) 'Ca II chromospheric emission and rotation of main sequence stars', Astron. Astrophys. 121, 190-197.

Duncan, D.K. (1981) 'Lithium abundances, K line emission, and ages of nearby solar type stars', Astrophys. J. 248, 651-669.

Durney, B.R. and Robinson, R.D. (1982) 'On an estimate of the dynamo-generated magnetic fields in late-type stars', Astrophys. J. 253, 290-297.

Gilliland, R.L. (1985) 'The relation of chromospheric activity to convection, rotation and evolution of the main sequence', Astrophys. J. 299, 286-294.

Giovanelli, R.G. (1980) 'An exploratory two-dimensional study of the coarse structure of network magnetic fields', Solar Phys. 68, 49-69. 
Noyes, R.W., Hartmann, L.W., Baliunas, S.L., Duncan, D.K., and Vaughan, A.H. (1984) 'Rotation, convection, and magnetic activity in lower main-sequence stars', Astrophys. J. 279, 763-777.

Rutten, R.G.M. (1987) 'Magnetic structure in cool stars. XII. Chromospheric activity and rotation of giants and dwarfs', Astron. Astrophys. 177, 131-142.

Schrijver, C.J. and Rutten, R.G.M. (1987) 'Magnetic structure in cool stars. XIV. Deficiency in chromospheric fluxes from M-type dwarfs', Astron. Astrophys. 177, 143-149.

Stauffer, J.R., Hartman, L., Soderblom, D.R., and Burnham, N. (1984) 'Rotational velocities of low-mass stars in the Pleiades', Astrophys. J. 280, 202-212.

Teplitskaya, R.B. (1989) 'On the relation between activity and rotation in the main-sequence stars', Astron. Nachr. 310, in press. 